\title{
El voluntariado corporativo en la estrategia de responsabilidad social de las empresas del IBEX 35
}

\section{Corporate volunteering within social responsibility strategies of IBEX 35 companies}

Dr. Luis Mañas-Viniegra es profesor e investigador de la Universidad Complutense de Madrid (España) (lmanas@ucm.es) (https://orcid.org/0000-0001-9129-5673)

\begin{abstract}
Resumen
El voluntariado corporativo se encuentra en auge en las empresas, que mejoran su reputación y el compromiso de los empleados con la política de responsabilidad social de la organización, a la vez que éstos contribuyen con su tiempo y habilidades a la mejora de las comunidades locales. Esta investigación analiza la implantación del voluntariado corporativo en las empresas del IBEX 35 a partir de un análisis de contenido de sus páginas web e informes anuales. El $25,71 \%$ de las empresas no tiene un programa de voluntariado corporativo declarado, a pesar de que en el sector Tecnología y Telecomunicaciones la implantación es del 100\%. El 45,71\% presenta un enfoque hacia su actividad empresarial y las acciones son principalmente sociales, educativas y medioambientales, incluyendo el 54,29\% los Objetivos de Desarrollo Sostenible (ODS) específicamente en sus acciones de voluntariado corporativo. Según la información recabada, existen pocos incentivos para los empleados y el 49,57\% de las empresas informa expresamente que no se podrá realizar voluntariado corporativo durante la jornada laboral. La comunicación comienza a ser específica, contando un $28,57 \%$ de las empresas con una marca de voluntariado corporativo ad hoc y un sitio web propio para fomentar la gestión y visibilidad de estos programas, a pesar de que sólo una empresa, Telefónica, dispone de redes sociales monotemáticas sobre su voluntariado corporativo, siendo también la única empresa que cuenta con una comisión de selección de voluntarios.
\end{abstract}

\begin{abstract}
Corporate volunteering is on the rise in companies as they strive to improve their reputation and the level of commitment of employees through policies of social responsibility. In turn, employees simultaneously contribute their time and skills to improving local communities. This research analyzes the implementation of corporate volunteering programs in IBEX 35 companies based on content analysis of their web pages and annual reports. The percentage of companies that do not have a corporate volunteering program is $25.71 \%$, despite the fact that the Technology and Telecommunications sector have a $100 \%$ implementation rate of such programs. Out of all IBEX 35 companies, $45.71 \%$ perform volunteering activities related to their business activity, and such actions are mainly social, educational and environmental. Moreover, from the companies on the IBEX 35, 54.29\% specifically include Sustainable Development Goals in their corporate volunteering actions. There are few incentives for employees, and $49.57 \%$ of companies expressly state that corporate volunteering activities cannot be carried out during working hours. Communication by companies of these volunteer programs has become more specific. As such, $28.57 \%$ of companies have a corporate network and their own website to promote the management and visibility of these programs, even if there is only one company, Telefónica, which offers social networks specifically committed to its corporate volunteering program, is the only company with a commission for selecting volunteers.
\end{abstract}

\section{Palabras clave I keywords}

Responsabilidad Social Corporativa, voluntariado corporativo, IBEX 35, voluntariado de empleados, conducta cívica organizacional, marca corporativa.

Corporate Social Responsibility, corporate volunteering, IBEX 35, employee volunteering, organizational citizenship behavior, corporate brand.

Cómo citar: Mañas-Viniegra, L. (2018). El voluntariado corporativo en la estrategia de responsabilidad social de las empresas del IBEX 35. Retos Revista de Ciencias de la Administración y Economía, 8(16), 19-32. https://doi.org/10.17163/ret.n16.2018.02 


\section{Introducción}

El voluntariado corporativo es la organización formal por parte de la empresa de programas para empleados que desean poner sus habilidades y su tiempo al servicio de la comunidad de forma voluntaria y desinteresada (Wild, 1993). Para ello, se desarrollan programas específicos en los que los empleados pueden participar como un equipo o se liberan horas de trabajo a cambio de participar individualmente en los programas de voluntariado de Organizaciones No Gubernamentales (ONG) en áreas como educación, salud, medioambiente, apoyo a jóvenes o personas mayores, entre otros (Solomon et al., 1991). El voluntariado corporativo es un fenómeno global en crecimiento (v. gr. Caligiuri, Mencin, \& Jiang, 2013; Rodell et al., 2016; Licandro, 2017) que hay que ubicar principalmente dentro de las empresas, puesto que el voluntariado corporativo dentro de las organizaciones sin ánimo de lucro se encuentra plenamente integrado con los propios servicios que prestan éstas (Roza et al., 2017), a pesar de que para las propias ONG como organizaciones sí existen posibilidades de colaboración con las empresas para organizar esos programas de voluntariado corporativo (Samuel, Wolf, \& Schiling, 2013).

El voluntariado corporativo hay que contextualizarlo dentro de la «economía de la reputación» (Johndrow, 2010) y de la creación de valor compartido (Porter, \& Kramer, 2011), en la que el propio trabajo se difumina como factor meramente económico y adquiere entidad como fenómeno sociocultural (Jones, 2011), deseando los empleados expresar valores significativos que refuerzan su integridad y que son inherentes a la actividad de voluntariado (Brockner, Senior, \& Welch, 2014).

Entre los motivos por los que los empleados realizan voluntariado corporativo destacan la lealtad a la organización, la oportunidad de mejorar la red de contactos, el desarrollo de habilidades y la satisfacción personal por la ayuda prestada (Loosemore, \& Bridgeman, 2017), aun reconociendo la influencia que la propia empresa puede tener en la decisión más allá de los beneficios personales que proporcionan las buenas acciones (Peloza, \& Hassay, 2006) y de los propios motivos altruistas (Peloza, Hudson, \& Hassay, 2009). Los empleados de menor edad son los más susceptibles de participar en el voluntariado corporativo (Peterson, 2004; Waikayi et al., 2012) debido a su compromiso inicial, a pesar de la necesidad de la motivación continua para la participación por parte de la empresa (Pajo, \& Lee, 2011). Además, las empleadas voluntarias muestran una mayor satisfacción laboral relacionada con el voluntariado, relación que no se muestra en el caso de los empleados de género masculino (Peterson, 2004a). Asimismo, la desigualdad de género se puso de manifiesto en Canadá al identificar menores probabilidades en las empleadas de recibir el apoyo de la empresa en tareas de voluntariado en forma de horas libres (MacPhail, \& Bowles, 2009).

Los principales beneficios del voluntariado corporativo para la organización que lo promueve son la reputación (Cycyota, Ferrante, \& Schroeder, 2016), la atracción del talento, el desarrollo de mayores habilidades (Jones, 2016) y la capacitación que adquieren los empleados (Loosemore, \& Bridgeman, 2017). En efecto, la atracción del talento hay que vincularla con el compromiso que los empleados adquieren con la misión de la empresa, en tanto que ésta comparte sus valores, ampliando su red de contactos con individuos que comparten similares valores en el entorno en el que trabajan (Bhattacharya, Sen, \& Korschun, 2008), considerando, además, que las iniciativas 
en responsabilidad social de una empresa mejoran la identificación del empleado con la empresa, su actitud, relación y comportamiento hacia la misma (Gilder et al., 2005; Muthuri, Matten, \& Moon, 2009; Kim et al., 2010). A pesar de esa mayor identificación con su empresa por parte de los voluntarios, no se ha asociado el voluntariado con mayores niveles de cumplimiento normativo o compliance en las empresas estadounidenses (Houghton, Gabel, \& Williams, 2009).

Es precisamente en un clima laboral de mutua confianza cuando los empleados ofrecen su creatividad y experiencia (Kim, \& Mauborgne, 1997). Las experiencias de voluntariado positivas que vayan acompañadas del apoyo de la dirección de la empresa fomentan que los empleados repitan su participación (Grant, 2012) e incrementen su lealtad a la organización (Carroll, 1990). A pesar de ello, no se puede obviar el componente de estrategia empresarial implícito en cualquier acción de responsabilidad social (Porter, \& Kramer, 2006) y la percepción positiva que ésta despierta en sus públicos estratégicos -stakeholders- (Plewa et al., 2015), lo que hace más propicio que sean las grandes empresas las que impulsen el voluntariado corporativo de un modo más formal, estratégico y generalizado que las pequeñas (Basil et al., 2011), aunque éstas deben mejorar la formación de los voluntarios, puesto que es el aspecto menos satisfactorio para ellos (Do-Paco, \& Nave, 2013).

Los programas de voluntariado corporativo también han comenzado a incorporar los Objetivos de Desarrollo Sostenible (ODS), que Naciones Unidas (2015) ha promovido entre las empresas como agentes activos para alcanzar los 17 objetivos marcados para el año 2030. A pesar de que tradicionalmente se ha considerado que la comunicación sobre las acciones en materia de responsabilidad social debía ser coherente con la actividad de la empresa, coherencia conocida como fit, comienza a ponerse en duda que se logre así una mayor convicción persuasiva sobre la honestidad de la organización (Villagra, Cárdaba, \& Ruiz San Román, 2016).

Más allá de la reputación que pueda suponer para la empresa o la implicación que ésta pueda lograr de sus empleados, hay que considerar diferentes tipos implantación en función de la dedicación horaria permitida por la organización. De este modo, un estudio realizado en Canadá puso de manifiesto que el respaldo pasivo al voluntariado se concretaba en tiempo libre no remunerado (71\%) y el ajuste de los horarios de trabajo (78\%), mientras que el respaldo activo con tiempo libre remunerado para destinar a la participación en el voluntariado se reducía al 29\% (Basil et al., 2009). Los voluntarios corporativos cuentan con el apoyo de familiares y amigos para ocupar su tiempo en estas tareas, pero su desempeño laboral puede estar sujeto a distracciones si su experiencia de voluntariado no supone un aprendizaje para ellos (Hu et al., 2016). Aunque no existen iniciativas normativas en este sentido, el Partido Conservador de Reino Unido incluyó en su programa electoral de 2015 la exigencia de que todas las grandes empresas y organismos públicos otorgaran tres días libres remunerados al año a sus empleados para participar en programas de voluntariado (Smith, 2017), pero la crisis que desencadenó el Brexit con la posterior dimisión del Primer Ministro, David Cameron, hizo que esta medida no llegase a proponerse formalmente.

En definitiva, el voluntariado corporativo ha ganado entidad dentro de los programas de responsabilidad social en las empresas, especialmente en las grandes, por 
lo que es necesario evaluar el grado de implantación en las empresas que cotizan en el IBEX 35 como referentes en España.

\section{Material y métodos}

La presente investigación busca determinar el grado de implantación del voluntariado corporativo dentro de la estrategia de responsabilidad social de las empresas del IBEX 35. Para ello, se servirá de los siguientes objetivos específicos: i) Establecer la implantación de programas de voluntariado corporativo por sectores empresariales del IBEX 35; ii) Determinar las empresas en las que estos programas se encuentran implantados y; iii) Recomendar buenas prácticas en cuanto a la implicación de los empleados en estos programas y la comunicación realizada a partir de las variables analizadas.

La hipótesis de partida $(\mathrm{HO})$ es que las principales empresas españolas todavía no han desarrollado sus programas de voluntariado corporativo en lo relativo a la implicación de sus empleados y especialmente en la comunicación e información pública dirigida a sus stakeholders.

La metodología se fundamenta en un análisis de contenido de las webs corporativas, informes de sostenibilidad y memorias de responsabilidad social de las empresas del IBEX 35 para identificar la información relativa al voluntariado corporativo que realizan. El análisis de contenido se ha realizado durante marzo de 2018 a partir del censo compuesto por las empresas que cotizan en el IBEX 35 al inicio de ese mes, agrupadas en los seis sectores que establece la Bolsa de Madrid (ver tabla 1).

Tabla 1. Empresas del IBEX 35 por sectores

\begin{tabular}{|l|l|}
\hline \multicolumn{1}{|c|}{ Sectores } & \multicolumn{1}{c|}{ Empresas } \\
\hline & Enagas \\
Petróleo y energía & Endesa \\
(Sector 1) & Gas Natural \\
& Iberdrola \\
& REE \\
& Repsol \\
\hline & Acciona \\
& Acerinox \\
Materiales básicos, industria y construcción & ACS \\
(Sector 2) & Arcelormittal \\
& Ferrovial \\
& Siemens Gamesa \\
\hline Bienes de consumo & Técnicas Reunidas \\
(Sector 3) & Grifols \\
\hline
\end{tabular}




\begin{tabular}{|l|l|}
\hline & Abertis \\
Servicios de consumo & AENA \\
(Sector 4) & DIA \\
& IAG \\
& Mediaset \\
& Meliá Hotels Intl. \\
\hline & Bankia \\
Servicios financieros e inmobiliarios & Bankinter \\
(Sector 5) & BBVA \\
& Caixa Bank \\
& Inmobiliaria Colonial \\
& Mapfre \\
& Merlin Properties \\
& Sabadell \\
Tecnología y telecomunicaciones & Santander \\
(Sector 6) & Amadeus \\
& Cellnex Telecom \\
& Indra \\
& Telefónica \\
\hline
\end{tabular}

El análisis de contenido se realiza a partir de tres categorías, integradas por 18 variables en total, emergentes a partir de una pre-revisión de dimensiones estructuradas en la revisión de la literatura científica y del análisis previo de una muestra piloto constituida por las 6 empresas cotizadas con mayor crecimiento en febrero de 2018 -mes inmediatamente anterior a la fecha del estudio-, en relación a la líder por cada sector referenciado en la tabla 1 . La relación de categorías y variables queda estructurada conforme al siguiente desglose (ver tabla 2):

Tabla 2. Categorías y variables de análisis

\begin{tabular}{|c|c|}
\hline Categorías & Variables \\
\hline Programa de voluntariado corporativo & $\begin{array}{l}\text { Responsabilidad de gestión a través de su } \\
\text { Fundación. } \\
\text { Colaboración con organizaciones sociales } \\
\text { profesionales. } \\
\text { Contempla los ODS. } \\
\text { Actividades relacionadas mayoritaria- } \\
\text { mente con su actividad empresarial. } \\
\text { Categoría de actividades ejecutadas. } \\
\text { Total participantes activos. } \\
\text { Comisión de selección de voluntarios. } \\
\text { Participantes ajenos a la organización. } \\
\mathrm{N}^{\circ} \text { de países. }\end{array}$ \\
\hline
\end{tabular}




\begin{tabular}{|l|l|}
\hline \multicolumn{1}{|c|}{ Categorías } & \multicolumn{1}{c|}{ Variables } \\
\hline \multirow{3}{*}{ Incentivos para los empleados } & Recompensas. \\
& $\begin{array}{l}\text { Premio voluntario del año. } \\
\text { Voluntariado en jornada de trabajo. } \\
\text { Gastos cubiertos en vacaciones solida- } \\
\text { rias. } \\
\text { Voluntariado online. } \\
\text { Programas internacionales. }\end{array}$ \\
\hline Comunicación del programa & Marca propia del programa. \\
& Web específica. \\
& Redes sociales específicas. \\
\hline
\end{tabular}

\section{Resultados}

A pesar del auge del voluntariado corporativo dentro de los planes de responsabilidad social en las empresas del IBEX 35, el 25,71\% de las empresas aún no dispone de un programa específico declarado para sus empleados y se limita a realizar meras aportaciones económicas a ONG u otras organizaciones a través de una estrategia más próxima al patrocinio y mecenazgo que a un plan de responsabilidad social. Los sectores «Petróleo y Energía» y «Tecnología y Telecomunicaciones» presentan voluntariado corporativo en el 100\% de las empresas de este índice bursátil español. Por el contrario, en el rubro «Servicios de consumo» únicamente el 33,33\% de las empresas ha implementado algún programa de este tipo.

El $28,57 \%$ afirma canalizar sus programas de voluntariado corporativo a través de sus fundaciones, incrementándose hasta el $57,14 \%$ en el sector de «Materiales Básicos», resultando sorprendente que un 54,29\% del total no ofrezca información en tal sentido. El 17,14\% confía la gestión de sus programas a departamentos específicos de la empresa, como es el caso de las entidades financieras BBVA -Área de Talento y Cultura- o Santander -Comité de Sostenibilidad-y se inicia la tendencia de combinar ambos modelos con la creación de comités formados por varios departamentos, como por ejemplo la empresa Gas Natural, que aglutina a Recursos Humanos, Comunicación y Medio Ambiente; e integrar la gestión de la fundación con un apoyo departamental, como es el caso de Endesa, Mapfre o Telefónica. Mención especial merece Caixa Bank, que ha constituido la Asociación de Voluntarios de La Caixa para gestionar íntegramente sus programas de voluntariado, entre los que se encuentra el corporativo.

Aunque el 54,29\% contempla los Objetivos de Desarrollo Sostenible (ODS) de forma expresa en su voluntariado corporativo, el $20 \%$ aún no los incluye, a pesar de que sí lo hacen dentro de sus objetivos de sostenibilidad en el ámbito medioambiental. En este sentido, todas las empresas del sector «Petróleo y Energía» los incorporan.

En relación al fit de las acciones de responsabilidad social que deben decidir las organizaciones en relación a su actividad, el $45,71 \%$ de las empresas enfocan mayoritariamente sus acciones de voluntariado corporativo hacia la propia actividad que ejercen, aunque ésta se adecúe a unos fines sociales. A pesar de ello, un 25,71\% esta- 
blece estas actividades en función de los públicos y las comunidades locales con las que interactúa, con una mayor presencia de actividades diversas.

«Servicios de Consumo» es el sector que menor dependencia tiene de su actividad, al orientar la temática de su voluntariado corporativo declarado (16,67\%). Estas actividades son mayoritariamente sociales $(65,71 \%)$, educativas, de formación o sensibilización $(57,14 \%)$, medioambientales $(40,00 \%)$, de inclusión $(34,29 \%)$, salud $(25,71 \%)$ y otras de diversa índole $(17,14 \%)$.

El número total de participantes activos declarados por las empresas del IBEX 35 en el último ejercicio asciende a 128 629. Dado que no todas han publicado los datos referidos al año 2017, a la fecha de finalización de la presente investigación no puede determinarse con exactitud qué porcentaje representa con respecto a los trabajadores actuales de las empresas del IBEX 35, pero se sitúa en torno al 10\%. El sector «Servicios Financieros e Inmobiliarios» aporta el mayor número de voluntarios activos, 75692 (58,84\% del total).

Únicamente una empresa, Telefónica, declara disponer de una comisión de selección de voluntarios, que establece unas directrices claras para esa selección, tales como: i) participación en otras actividades del programa, ii) conocimiento de la acción social de la compañía, iii) experiencia en otros proyectos sociales, iv) adecuación del perfil a la actividad y, v) la motivación expresada para la participación. El resto de las empresas no ofrece información alguna al respecto, lo que permite inferir que el único criterio de selección es el orden de inscripción en el programa hasta completar el cupo de plazas disponibles. Este criterio aparece reflejado en la mayoría de los programas, pero no se indica si es el único excluyente, además de los que establece la propia Ley de Voluntariado (Ley 45/2015, de 14 de octubre), como no estar incluido en el registro de delincuentes sexuales y otros similares.

Sólo el 14,28\% de las empresas permite a participantes ajenos a la organización, es decir, empleados, prejubilados y jubilados, inscribirse en las acciones de voluntariado corporativo, aunque de manera puntual los familiares pueden hacerlo en jornadas que suelen duran un día y tienen un carácter de concienciación e incluso convivencia, más que de participación activa. A pesar de ello, alguna empresa como AENA permite participar a proveedores y otras, como Caixa Bank o Indra, hacen lo propio con los clientes.

Los programas de voluntariado corporativo también son acordes a la vocación multinacional de las empresas del IBEX 35, extendiéndose tales programas a un total de 214 países agregados, muchos de ellos relacionados con la actividad de cada empresa y otros, determinados por la propia urgencia social de los países en los que se desarrollan. 
Tabla 3. Análisis de los programas de voluntariado corporativo

\begin{tabular}{|c|c|c|c|c|c|c|c|c|c|c|c|c|c|c|}
\hline $\begin{array}{l}\text { Programa de } \\
\text { voluntariado }\end{array}$ & \multicolumn{2}{|c|}{ Sector $1(\%)$} & \multicolumn{2}{|c|}{ Sector $2(\%)$} & \multicolumn{2}{|c|}{ Sector $3(\%)$} & \multicolumn{2}{|c|}{ Sector $4(\%)$} & \multicolumn{2}{|c|}{ Sector $5(\%)$} & \multicolumn{2}{|c|}{$\begin{array}{c}\text { Sector } 6 \\
(\%)\end{array}$} & \multicolumn{2}{|c|}{$\begin{array}{l}\text { Total } \\
(\%)\end{array}$} \\
\hline \multirow{3}{*}{$\begin{array}{l}\text { Responsabili- } \\
\text { dad de gestión } \\
\text { a través de su } \\
\text { Fundación }\end{array}$} & Sí & 33,33 & Sí & 57,14 & Sí & 0,00 & Sí & 16,67 & Sí & 22,22 & Sí & 25,00 & Sí & 28,57 \\
\hline & No & 16,67 & No & 0,00 & No & 66,67 & No & 0,00 & No & 33,33 & No & 0,00 & No & 17,14 \\
\hline & N/D & 50,00 & N/D & 42,86 & N/D & 33,33 & N/D & 83,33 & N/D & 44,44 & N/D & 75,00 & N/D & 54,29 \\
\hline \multirow{3}{*}{$\begin{array}{l}\text { Colaboración } \\
\text { con organiza- } \\
\text { ciones sociales } \\
\text { profesionales }\end{array}$} & Sí & 100,00 & Sí & 71,43 & Sí & 66,67 & Sí & 33,33 & Sí & 77,78 & Sí & 75,00 & Sí & 71,43 \\
\hline & No & 0,00 & No & 0,00 & No & 0,00 & No & 0,00 & No & 0,00 & No & 0,00 & No & 0,00 \\
\hline & N/D & 0,00 & N/D & 28,57 & N/D & 33,33 & N/D & 66,67 & N/D & 22,22 & N/D & 25,00 & N/D & 28,57 \\
\hline \multirow{3}{*}{$\begin{array}{l}\text { Contempla los } \\
\text { ODS }\end{array}$} & Sí & 100,00 & Sí & 42,86 & Sí & 33,33 & Sí & 33,33 & Sí & 44,44 & Sí & 75,00 & Sí & 54,29 \\
\hline & No & 0,00 & No & 28,57 & No & 33,33 & No & 0,00 & No & 33,33 & No & 25,00 & No & 20,00 \\
\hline & N/D & 0,00 & N/D & 28,57 & N/D & 33,33 & N/D & 66,67 & N/D & 22,22 & N/D & 0,00 & N/D & 25,71 \\
\hline \multirow{3}{*}{$\begin{array}{l}\text { Actividades } \\
\text { relacionadas } \\
\text { mayoritaria- } \\
\text { mente con } \\
\text { su actividad } \\
\text { empresarial }\end{array}$} & Sí & 83,33 & Sí & 42,86 & Sí & 33,33 & Sí & 16,67 & Sí & 33,33 & Sí & 75,00 & Sí & 45,71 \\
\hline & No & 16,67 & No & 28,57 & No & 33,33 & No & 0,00 & No & 44,44 & No & 25,00 & No & 25,71 \\
\hline & N/D & 0,00 & N/D & 28,57 & N/D & 33,33 & N/D & 83,33 & N/D & 22,22 & N/D & 0,00 & N/D & 28,57 \\
\hline \multirow{6}{*}{$\begin{array}{l}\text { Categoría de } \\
\text { actividades } \\
\text { ejecutadas } \\
\text { (Social = SL; } \\
\text { Educación = } \\
\text { ED; Medio } \\
\text { ambiente = MA; } \\
\text { Salud = SD; } \\
\text { Inclusión = IN; } \\
\text { Otras = OT) }\end{array}$} & $\mathrm{SL}$ & 100,00 & SL & 57,14 & SL & 66,67 & SL & 16,67 & SL & 66,67 & SL & 100,00 & SL & 65,71 \\
\hline & ED & 100,00 & ED & 71,43 & ED & 33,33 & ED & 0,00 & ED & 66,67 & ED & 50,00 & ED & 57,14 \\
\hline & MA & 83,33 & MA & 28,57 & MA & 0,00 & MA & 0,00 & MA & 44,44 & MA & 75,00 & MA & 40,00 \\
\hline & SD & 33,33 & $\mathrm{SD}$ & 28,57 & SD & 33,33 & SD & 16,67 & SD & 22,22 & SD & 25,00 & SD & 25,71 \\
\hline & IN & 66,67 & IN & 0,00 & IN & 33,33 & IN & 0,00 & IN & 55,56 & IN & 50,00 & IN & 34,29 \\
\hline & OT & 33,33 & OT & 28,57 & OT & 0,00 & OT & 16,67 & OT & 0,00 & OT & 25,00 & OT & 17,14 \\
\hline $\begin{array}{l}\text { Total partici- } \\
\text { pantes activos }\end{array}$ & \multicolumn{2}{|c|}{6.462} & \multicolumn{2}{|c|}{15.135} & \multicolumn{2}{|c|}{1.300} & \multicolumn{2}{|l|}{ N/D } & \multicolumn{2}{|c|}{75.692} & \multicolumn{2}{|c|}{30.040} & \multicolumn{2}{|c|}{128.629} \\
\hline \multirow{3}{*}{$\begin{array}{l}\text { Comisión de } \\
\text { selección de } \\
\text { voluntarios }\end{array}$} & Sí & 0,00 & Sí & 0,00 & Sí & 0,00 & Sí & 0,00 & Sí & 0,00 & Sí & 25,00 & Sí & 2,86 \\
\hline & No & 0,00 & No & 0,00 & No & 0,00 & No & 0,00 & No & 0,00 & No & 0,00 & No & 0,00 \\
\hline & N/D & 100,00 & N/D & 100,00 & N/D & 100,00 & N/D & 100,00 & N/D & 100,00 & $\mathrm{~N} / \mathrm{D}$ & 75,00 & N/D & 97,14 \\
\hline \multirow{3}{*}{$\begin{array}{l}\text { Participantes } \\
\text { ajenos a la } \\
\text { organización }\end{array}$} & Sí & 16,67 & Sí & 0,00 & Sí & 0,00 & Sí & 16,67 & Sí & 22,22 & Sí & 25,00 & Sí & 14,28 \\
\hline & No & 50,00 & No & 14,29 & No & 0,00 & No & 0,00 & No & 55,56 & No & 50,00 & No & 31,43 \\
\hline & N/D & 33,33 & N/D & 85,71 & N/D & 100,00 & N/D & 83,33 & N/D & 22,22 & N/D & 25,00 & N/D & 54,29 \\
\hline $\begin{array}{l}\mathrm{N}^{\circ} \text { de países } \\
\text { (inc.España) }\end{array}$ & & 21 & & 58 & & 10 & & 1 & & 34 & & 90 & & 214 \\
\hline
\end{tabular}

Ninguna empresa declara en su información pública ofrecer días libres adicionales por participar en jornadas de voluntariado corporativo, más allá de que la participación del empleado se produzca durante la jornada laboral, algo que sucede únicamente en el $22,86 \%$ de los casos, declarando expresamente el $48,57 \%$ que no se contempla esta posibilidad. Únicamente Telefónica reconoce la labor del voluntario corporativo con un premio específico para esta labor, cubriendo además todos los gastos de los empleados 
que se acogen al programa de vacaciones solidarias junto a Iberdrola, las dos únicas empresas que lo hacen. Sorprende también que, a pesar del carácter multinacional de las empresas del IBEX 35, apenas el $45,71 \%$ de sus programas de voluntariado tengan un carácter internacional, en el sentido de que los empleados de un centro de trabajo en un país puedan participar en las actividades organizadas en los demás países por los otros centros. Tampoco se encuentran desarrollados los programas de voluntariado online, impulsados sólo por el 14,29\% de las empresas, todas ellas del sector energético junto a Telefónica, que destaca especialmente en este apartado.

Tabla 4. Análisis de los incentivos para empleados del voluntariado corporativo

\begin{tabular}{|c|c|c|c|c|c|c|c|c|c|c|c|c|c|c|}
\hline \multirow{4}{*}{\begin{tabular}{l}
\multicolumn{1}{c}{$\begin{array}{c}\text { Incentivos } \\
\text { para los } \\
\text { empleados }\end{array}$} \\
$\begin{array}{l}\text { Recompensas } \\
\text { (días libres } \\
\text { extras) }\end{array}$
\end{tabular}} & \multicolumn{2}{|c|}{$\begin{array}{c}\text { Sector } 1 \\
(\%)\end{array}$} & \multicolumn{2}{|c|}{$\begin{array}{c}\text { Sector } 2 \\
(\%)\end{array}$} & \multicolumn{2}{|c|}{$\begin{array}{c}\text { Sector } 3 \\
(\%)\end{array}$} & \multicolumn{2}{|c|}{$\begin{array}{l}\text { Sector } 4 \\
(\%)\end{array}$} & \multicolumn{2}{|c|}{ Sector $5(\%)$} & \multicolumn{2}{|c|}{$\begin{array}{c}\text { Sector } 6 \\
(\%)\end{array}$} & \multicolumn{2}{|c|}{$\begin{array}{l}\text { Total } \\
(\%)\end{array}$} \\
\hline & Sí & & Sí & 0,00 & Sí & 0,00 & Sí & & Sí & 0,00 & Sí & 0,00 & Sí & 0,00 \\
\hline & & 100,00 & No & 71,43 & No & 66,67 & No & 0,00 & No & 0,00 & No & 100,00 & No & 48,57 \\
\hline & & 0,00 & N/D & 28,57 & N/D & 33,33 & N/D & 100,00 & N/D & 100,00 & N/D & 0,00 & N/D & 51,43 \\
\hline \multirow{3}{*}{$\begin{array}{l}\text { Premio volunta- } \\
\text { rio del año }\end{array}$} & Sí & 0,00 & Sí & 0,00 & Sí & 0,00 & Sí & 0,00 & Sí & 0,00 & Sí & 25,00 & Sí & 2,86 \\
\hline & N & 100,00 & No & 71,43 & No & 66,67 & No & 16,67 & No & 77,78 & No & 75,00 & No & 68,57 \\
\hline & N/D & 0,00 & N/D & 28,57 & N/D & 33,33 & N/D & 83,33 & N/D & 22,22 & N/D & 0,00 & N/D & 28,57 \\
\hline \multirow{3}{*}{$\begin{array}{l}\text { Voluntariado en } \\
\text { jornada de tra- } \\
\text { bajo }\end{array}$} & & & Sí & 28,57 & Sí & 33,33 & Sí & 0,00 & Sí & 11,11 & Sí & 50,00 & Sí & 22,86 \\
\hline & No & 16,67 & No & 0,00 & No & 0,00 & No & 0,00 & No & 0,00 & No & 0,00 & No & 2,86 \\
\hline & N/D & 50,00 & N/D & 71,43 & N/D & 66,67 & N/D & 100,00 & N/D & 88,89 & N/D & 50,00 & N/D & 74,29 \\
\hline \multirow{3}{*}{$\begin{array}{l}\text { Gastos cubiertos } \\
\text { en vacaciones } \\
\text { solidarias }\end{array}$} & Sí & 16,67 & Sí & 14,29 & Sí & 0,00 & Sí & 0,00 & Sí & 0,00 & Sí & 25,00 & Sí & 8,57 \\
\hline & No & 0,00 & No & 0,00 & No & 0,00 & No & 0,00 & No & 0,00 & No & 0,00 & No & 0,00 \\
\hline & N/D & 83,33 & N/D & 85,71 & N/D & 100,00 & N/D & 100,00 & N/D & 100,00 & N/D & 75,00 & N/D & 91,43 \\
\hline \multirow{3}{*}{$\begin{array}{l}\text { Voluntariado } \\
\text { online }\end{array}$} & Sí & 50,00 & Sí & 0,00 & Sí & 0,00 & Sí & 0,00 & Sí & 0,00 & Sí & 50,00 & Sí & 14,29 \\
\hline & No & 50,00 & No & 71,43 & No & 66,67 & No & 16,67 & No & 77,78 & No & 25,00 & No & 54,29 \\
\hline & N/D & 0,00 & N/D & 28,57 & N/D & 33,33 & N/D & 83,33 & N/D & 22,22 & N/D & 25,00 & N/D & 31,43 \\
\hline \multirow{3}{*}{$\begin{array}{l}\text { Programas in- } \\
\text { ternacionales }\end{array}$} & Sí & 66,67 & Sí & 71,43 & Sí & 66,67 & Sí & 0,00 & Sí & 22,22 & Sí & 75,00 & Sí & 45,71 \\
\hline & No & 33,33 & No & 0,00 & No & 0,00 & No & 16,67 & No & 22,22 & No & 0,00 & No & 14,29 \\
\hline & N/D & 0,00 & N/D & 28,57 & N/D & 33,33 & N/D & 83,33 & N/D & 55,56 & N/D & 25,00 & N/D & 40,00 \\
\hline
\end{tabular}

El 28,57\% de las empresas dispone ya de una marca propia de voluntariado corporativo que va más allá de incluir la palabra «voluntarios» a la marca corporativa e implica un logo propio acorde con la identidad visual corporativa o un naming específico. Nombres tan diversos como «EnREDando», «Solidarios», «Altruis», "Mueve.te» o «Sabadell Life», se acompañan con logos específicos como los que pueden apreciarse en la siguiente figura:

Figura 1.Identidad visual corporativa de los programas de voluntariado corporativo
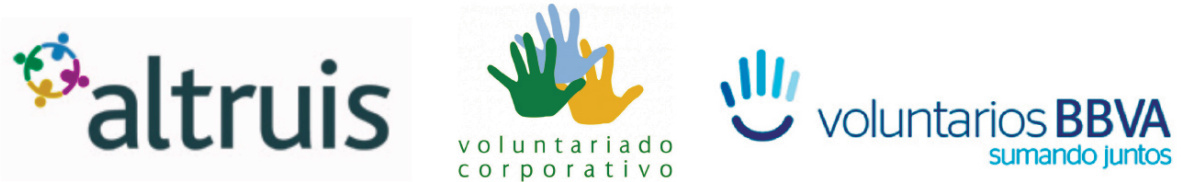

Fuente: webs corporativas de las empresas (2018) 
Precisamente, de las empresas que disponen de una marca propia para su programa de voluntariado corporativo, el 28,57\% también han desarrollado una web específica para gestionar y dar visibilidad a sus programas, en muchas de las cuales se integran los programas de voluntariado de las ONG con las que colaboran y los propios programas de voluntariado corporativo. Menor aún es la presencia en redes sociales con cuentas y contenidos referidos específicamente a los programas de voluntariado corporativo y únicamente Telefónica lo hace en Facebook, Twitter, Instagram y Youtube. El seguimiento por parte de los públicos es escaso, alcanzando 8398 likes en su fan page de Facebook. El resto de empresas opta por integrar la comunicación de sus acciones de voluntariado como una publicación más dentro de las redes sociales de sus fundaciones o de la propia empresa, aunque alguna como Mapfre lo hace con el hashtag propio \#VoluntariosMAPFRE. Llama la atención que Caixa Bank, que es una de las empresas con un programa de voluntariado corporativo más desarrollado, utilice Facebook como grupo privado, es decir, como herramienta de comunicación interna y no externa.

Tabla 5. Análisis de la comunicación del voluntariado corporativo

\begin{tabular}{|c|c|c|c|c|c|c|c|c|c|c|c|c|c|c|}
\hline \multirow{4}{*}{$\begin{array}{l}\begin{array}{l}\text { Comunicación } \\
\text { del programa }\end{array} \\
\begin{array}{l}\text { Marca propia } \\
\text { del programa }\end{array}\end{array}$} & \multicolumn{2}{|c|}{ Sector $1(\%)$} & \multicolumn{2}{|c|}{ Sector $2(\%)$} & \multicolumn{2}{|c|}{ Sector $3(\%)$} & \multicolumn{2}{|c|}{ Sector $4(\%)$} & \multicolumn{2}{|c|}{ Sector $5(\%)$} & \multicolumn{2}{|c|}{ Sector $6(\%)$} & \multicolumn{2}{|c|}{ Total (\%) } \\
\hline & Sí & & Sí & 0,00 & Sí & 33,33 & Sí & 16,67 & Sí & 55,56 & Sí & 0,00 & Sí & 28,57 \\
\hline & No & 50,00 & No & 71,43 & No & 33,33 & No & 16,67 & No & 22,22 & No & 100,00 & No & 45,71 \\
\hline & & 0,00 & N/D & 28,57 & N/D & & N/D & & N/D & 22,22 & N/D & 0,00 & N/D & 25,71 \\
\hline \multirow{3}{*}{ Web específica } & Sí & & Sí & 0,00 & Sí & 33,33 & Sí & 0,00 & Sí & 55,56 & Sí & 25,00 & Sí & 28,57 \\
\hline & No & 50,00 & No & 71,43 & No & 33,33 & No & 33,33 & No & 22,22 & No & 75,00 & No & 45,71 \\
\hline & N/D & 0,00 & N/D & 28,57 & N/D & 33,33 & N/D & 66,67 & N/D & 22,22 & $\mathrm{~N} / \mathrm{D}$ & 0,00 & N/D & 25,71 \\
\hline \multirow{3}{*}{$\begin{array}{l}\text { Redes sociales } \\
\text { específicas }\end{array}$} & Sí & 0,00 & Sí & 0,00 & Sí & 0,00 & Sí & 0,00 & Sí & 0,00 & Sí & 25,00 & Sí & 2,86 \\
\hline & No & 100,00 & No & 71,43 & No & 66,67 & No & 33,33 & No & 77,78 & No & 75,00 & No & 71,43 \\
\hline & N/D & 0,00 & N/D & 28,57 & N/D & 33,33 & N/D & 66,67 & N/D & 22,22 & N/D & 0,00 & N/D & 25,71 \\
\hline
\end{tabular}

\section{Discusión y conclusiones}

La mayoría de las empresas cotizadas en el índice bursátil español, IBEX 35, presenta programas de voluntariado corporativo, sin embargo son muchas las que lo tienen poco definido y confían totalmente su gestión a organizaciones externas, algo que sólo tiene sentido en una fase primigenia de desarrollo del mismo y que se encuentra más próximo al voluntariado que al voluntariado corporativo como apartado de la gestión estratégica.

Que más de la mitad de las empresas no ofrezcan información sobre si es la fundación (todas las empresas del IBEX 35 cuentan con una fundación generada a partir de su actividad) la última responsable de la gestión del programa de voluntariado corporativo, hace sospechar que se intenta evitar la sensación de que estos programas se pongan en marcha con una finalidad meramente fiscal, lo cual es acertado en términos de comunicación, pero escasamente coherente en términos de responsabilidad social, en tanto que se despejaría cualquier duda al canalizarlo a través de un departamento específico o un comité dentro de la empresa relacionado con la dirección de personas, la acción social, la comunicación, y la sostenibilidad, que, por otra parte, contribuiría a profesionalizar y personalizar el voluntariado corporativo dentro de la empresa. 
La integración de los ODS dentro de los programas de voluntariado corporativo implica reconocer su contribución y la de los empleados participantes al reto marcado por Naciones Unidas para 2030 y comienza a desaparecer el reduccionismo que supone limitar estos OSD a las actuaciones de gestión medioambiental que venían haciéndose a través de los planes de sostenibilidad, dotándolos de un alcance global y transversal dentro de la organización.

Asimismo, la mitad de las empresas del IBEX 35 adoptan un enfoque más táctico que estratégico a la hora de decidir el «encaje» o fit de las actividades de voluntariado corporativo con su propia actividad como organización, lo que refleja también que todavía nos encontramos en las primeras fases de desarrollo de estos programas dentro de la política de responsabilidad social en España.

A pesar del avance en el número de empleados que participan activamente en programas de voluntariado corporativo, el 10\% aún es una cifra que permite inferir el recorrido prospectivo de mejora que todavía deben implementar estos programas. También cabe poner en duda que esas cifras sean las reales, pues en determinada información proporcionada por algunas empresas no se refleja claramente que los empleados declarados sean los que realizan voluntariado corporativo, excluyendo una mera aportación económica que se realiza, por ejemplo, durante el Día Internacional del Voluntariado en el que muchas empresas participan. Podría, por tanto, ser inferior la participación de manera estricta en el voluntariado corporativo.

A pesar de que sólo Telefónica cuenta con la comisión de selección de voluntarios, lo que implica una auténtica profesionalización de estos programas y de la máxima implicación en su buen desarrollo por parte de la empresa, hay que considerar que, analizando la agenda de actividades de voluntariado de las diferentes empresas, muchas de éstas no alcanzan el objetivo en cuanto al número máximo de inscritos en el que se situaba el límite, lo que explica -que no justifica-, por qué aún no es una cuestión prioritaria para las demás empresas.

La apertura de los programas de voluntariado corporativo a clientes y proveedores, a pesar de su escasa implantación, pueden resultar una iniciativa interesante desde el punto de vista de integración de un mayor número de stakeholders con los que los empleados tienen un trato directo en sus funciones profesionales, pudiendo favorecer una mejor relación en su participación en programas de corte altruista, alejados de la tensión que la actividad económica puede despertar en el día a día de esa relación proveedor-cliente.

Aunque la propia esencia del voluntariado presupone una elección altruista que no espera a cambio más recompensa que la satisfacción personal, la implicación de la empresa que pone en marcha un programa de voluntariado corporativo debe plasmar el máximo compromiso favoreciendo la participación y reconociendo la labor de sus empleados.

Debe establecer un mínimo de horas de voluntariado que pueden realizarse durante la jornada laboral, algo que apenas sucede todavía, sin más limitación que las necesidades de servicio inexcusables que pudieran producirse en las fechas de la actividad para el normal funcionamiento de la empresa. Lo que no puede hacer es limitarla al tiempo libre o a las vacaciones de los empleados, ya que, en este caso, el voluntariado deja de ser corporativo y se convierte ipso facto en altruismo de los empleados. 
Sin embargo, en el caso contrario, otorgar días libres adicionales como recompensa por haber participado como voluntario corporativo implicaría contravenir la Ley de Voluntariado (Ley 45/2015, de 14 de octubre), que expresa claramente en su artículo 3 que se llevarán «a cabo sin contraprestación económica o material, sin perjuicio del abono de los gastos reembolsables que el desempeño de la acción voluntaria ocasione a los voluntarios».

Cubrir totalmente los gastos de los empleados en los programas de vacaciones solidarias, potenciar el carácter internacional de los mismos, ya que sus empleados tienen un perfil eminentemente internacional, e implantar premios propios de voluntariado y programas online para los empleados que por sus propias funciones no puedan ausentarse de su puesto de trabajo para participar en otro tipo de programas son otras decisiones que deberían adoptar las empresas para fortalecer su voluntariado corporativo.

Estando en auge la creación de marcas y sitios web específicos de voluntariado corporativo para la gestión de los programas y la obtención de la visibilidad adecuada, sólo Telefónica dispone de redes sociales específicas para estos programas, lo que permite inferir que la comunicación de voluntariado corporativo sigue concibiéndose como una acción más integrada dentro de los planes de responsabilidad social o de las acciones de la fundación derivada de la empresa, a pesar de que algunas empresas identifiquen con un hashtag propio este tipo de programas en su comunicación externa.

Consecuentemente, los programas de voluntariado corporativo en las empresas del IBEX 35 aún deben seguir implantándose y desarrollándose en aquéllas que ya los han consolidando con una gestión directa y propia, mejorando la participación de los empleados a través de los incentivos y ampliando la comunicación realizada.

Las limitaciones del estudio se centran en que el análisis de contenido se ha realizado en un momento concreto de estos programas de voluntariado corporativo, por lo que las futuras líneas de investigación deben abordar la evolución en comparación con lo realizado en empresas estadounidenses por la anticipación que muestran en la implantación de acciones responsables y la percepción que los públicos y los propios empleados tienen sobre estos programas, determinando el grado de repercusión en la reputación de la marca corporativa.

\section{Referencias}

Basil, D. Z., Runte, M., Basil, M., \& Usher, J. (2011). Company support for employee volunteerism Does size matter? Journal of Business Research, 64(1), 61-66. https://doi.org/10.1016/j. jbusres.2009.10.002

Basil, D. Z., Runte, M. S., Easwaramoorthy, M., \& Barr, C. (2009). Company Support for Employee Volunteering: A National Survey of Companies in Canada. Journal of Business Ethics, 85, 387-398. https://doi.org/10.1007/s10551-008-9741-0

Bhattacharya, C. B., Sen, S., \& Korschun, D. (2008). Using corporate social responsibility to win the war for talent. MIT Sloan Management Review, 49(2), 37-44. goo.gl/at1uHM

Brockner, J., Senior, D., \& Welch, W. (2014). Corporate Volunteerism, the Experience of Self-Integrity, and Organizational Commitment: Evidence from the Field. Social Justice Research, 27(1), 1-23. https://doi.org/10.1007/s11211-014-0204-8 
Caligiuri, P., Mencin, A., \& Jiang, K. F. (2013). Win-win-win: the influence of company-sponsored volunteerism programs on employees, NGOs, and business units. Personnel Psychology, 66(4), 825-860. https://doi.org/10.1111/peps.12019

Carroll, P. B. (1990, 10 de agosto). Chivas Regal Report on working Americans: emerging values for the 1990s. Wall Street Journal, B6.

Cycyota, C. S., Ferrante, C. J., \& Schroeder, J. M. (2016). Corporate social responsibility and employee volunteerism: What do the best companies do? Business Horizons, 59(3), 321-329. https://doi.org/10.1016/j.bushor.2016.01.004

Do-Paco, O., \& Nave, A. C. (2013). Corporate volunteering A case study centred on the motivations, satisfaction and happiness of company employees. Employee Relations, 35(5), 547559. https://doi.org/10.1108/ER-12-2012-0089

Gilder, D., Schuyt, T. N. M., \& Breedijk, M. (2005). Effects of an employee volunteering program on the work force: The ABN-AMRO case. Journal of Business Ethics, 61(2), 143-152. https:// doi.org/10.1007/s10551-005-7101-x

Grant, A. M. (2012). Giving time, time after time: work design and sustained employee participation in corporate volunteering. Academy of Management Review, 37(4), 589-615. https://doi. org/10.5465/amr.2010.0280

Houghton, S., Gabel, J. T. A., \& Williams, D. W. (2009). Connecting the Two Faces of CSR: Does Employee Volunteerism Improve Compliance? Journal of Business Ethics, 87(4), 477-494. https://doi.org/10.1007/s10551-008-9954-2

Hu, J., Jiang, K. F., Mo, S. J., Chen, H. H., \& Shi, J. Q. (2016). The motivational antecedents and performance consequences of corporate volunteering: When do employees volunteer and when does volunteering help versus harm work performance? Organizational Behavior and Human Decision Processes, 137, 99-111. https://doi.org/10.1016/j.obhdp.2016.08.005

Kim, H. R., Lee, M., Lee, H. T., \& Kim, N. M. (2010). Corporate Social Responsibility and Employee-Company Identification. Journal of Business Ethics, 95(4), 557-569. https://doi. org/10.1007/s10551-010-0438-9

Kim, W. C., \& Mauborgne, R. (1997). Fair process: Managing in the knowledge economy. Harvard Business Review, 75(4), 65-75. goo.gl/qcBXbt

Johndrow, A. (2010, 27 de agosto). Making money in the reputation economy. Forbes. goo.gl/ qwDnUO

Jones, A. (2011). Theorising international youth volunteering: training for global (corporate) work? Transactions of the Institute of British Geographers, 36(4),530-544. https://doi.org/10.1111/ j.1475-5661.2011.00433.x

Jones, D. A. (2016). Widely Assumed but Thinly Tested: Do Employee Volunteers' Self-Reported Skill Improvements Reflect the Nature of Their Volunteering Experiences? Frontiers in Psychology, 7, 1-24. https://doi.org/10.3389/fpsyg.2016.00495

Ley 45/2015, de 14 de octubre, de Voluntariado. BOE, 247, de 15 de octubre de 2015. (goo.gl/ XmPLT7).

Licandro, O. (2017). The relationship between corporate volunteering and corporate social responsibility: results of an empirical study. Ekonomski Vjesnik, 30(1), 67-83. (goo.gl/ViSBNN).

Loosemore, M., \& Bridgeman, J. (2017). Corporate volunteering in the construction industry: motivations, costs and benefits. Construction Management and Economics, 35(10), 641-653. https://doi.org/10.1080/01446193.2017.1315150

MacPhail, F., \& Bowles, P. (2011). Corporate Social Responsibility as Support for Employee Volunteers: Impacts, Gender Puzzles and Policy Implications in Canada. Journal of Business Ethics, 84(3), 405-416. https://doi.org/10.1007/s10551-008-9716-1

Muthuri, J. N., Matten, D., \& Moon, J. (2009). Employee Volunteering and Social Capital: Contributions to Corporate Social Responsibility. British Journal of Management, 20(1), 75-89. https://doi.org/10.1111/j.1467-8551.2007.00551.x

Naciones Unidas (2015). Objetivos de desarrollo sostenible. goo.gl/3cGMLL 
Pajo, K., \& Lee, L. (2011). Corporate-Sponsored Volunteering: A Work Design Perspective. Journal of Business Ethics, 99(3), 467-482. https://doi.org/10.1007/s10551-010-0665-0

Peloza, J., \& Hassay, D. N. (2006). Intra-organizational volunteerism: Good soldiers, good deeds and good politics. Journal of Business Ethics, 64(4), 357-379. https://doi.org/10.1007/s10551005-5496-Z

Peloza, J., Hudson, S., \& Hassay, D. N. (2009). The Marketing of Employee Volunteerism. Journal of Business Ethics, 85, 371-386. https://doi.org/10.1007/s10551-008-9734-z

Peterson, D. K. (2004). Recruitment strategies for encouraging participation in corporate volunteer programs. Journal of Business Ethics, 49(4), 371-386. https://doi.org/10.1023/B:BUSI.0000020872.10513.f2

Peterson, D. K. (2004a). Benefits of participation in corporate volunteer programs: employees' perceptions. Personnel Review, 33(5-6), 615-627. https://doi.org/10.1108/00483480410561510

Plewa, C., Conduit, J., Quester, P. G., Pascale, G., \& Johnson, C. (2015). The Impact of Corporate Volunteering on CSR Image: A Consumer Perspective. Journal of Business Ethics, 127(3), 643 659. https://doi.org/10.1007/s10551-014-2066-2

Porter M. E., \& Kramer, M. R. (2006). Strategy and society: the link between competitive advantage and corporate social responsibility. Harvard Business Review, 84(12), 78-92.

Porter, M. E., \& Kramer, M. R. (2011). Creating shared value. Harvard Business Review, 84(12), 42-56.

Rodell, J. B., Breitsohl, H., Schroeder, M., \& Keating, D. J. (2016). Employee Volunteering: A Review and Framework for Future Research. Journal of Management, 42(1), 55-84. https:// doi.org/10.1177/0149206315614374

Roza, L., Shachar, I., Mejis, L., \& Hustinx, L. (2017). The nonprofit case for corporate volunteering: a multi-level perspective. Service Industries Journal, 37(11-12), 746-765. https://doi.org/ 10.1080/02642069.2017.1347158

Samuel, O., Wolf, P., \& Schilling, A. (2013). Corporate Volunteering Benefits and Challenges for Nonprofits. Nonprofit Management $\mathcal{E}$ Leadership, 24(2), 163-179. https://doi.org/10.1002/ $\mathrm{nml} .21089$

Smith, J. D. (2017). David Cameron's three-day volunteering initiative: genuine proposal or electoral sham? Voluntary Sector Review, 8(2), 219-227. https://doi.org/10.1332/20408051 7X14938215340094

Solomon, S. W., Ragland, B. O., Wilson, R. E., \& Plost, M. (1991). Encouraging company employees to volunteer. Corporate Contribution. En J.P. Shannon (ed.), The Corporate Contributions Handbook. San Francisco: Jossey-Bass.

Villagra, N., Cárdaba, M. A. M., \& Ruiz San Román, J. A. (2016). Comunicación de RSC: una revisión de las tesis clásicas sobre la coherencia entre la acción de RSC y la actividad organizacional. Communication $\mathcal{E}$ Society, 29(2), 133-149. https://doi.org/10.15581/003.29.2.sp.133-149

Waikayi, L., Fearon, C., Morris, L., \& McLaughlin, H. (2012). Volunteer management: an exploratory case study within the British Red Cross. Management Decision, 50(3-4), 349-367. https://doi.org/10.1108/00251741211216188

Wild, C. (1993). Corporate Volunteer Programs: Benefits to Business (The Conference Board, New York, NY) Report 1029. 